\title{
Pathophysiology of schizophrenia causal role for dopamine or noradrenaline?
}

from L. L. Iversen, G. P. Reynolds and S. H. Snyder

IN a recent article in Nature, Hornykiewicz ${ }^{1}$ has provided a thorough and thoughtful review of the abundant literature on the role of catecholamines in schizophrenia and advocated a role for noradrenaline, while criticizing evidence for dopamine involvement. In discussions of catecholamines in schizophrenia, all too often two separate issues are muddled together. One is the role of various neurotransmitters in mediating the therapeutic effects of drugs in schizophrenia; the other is the possible alterations in neurotransmitters or their regulatory enzymes in schizophrenic brain. The evidence on the former issue is strong, that on the latter weaker.

Much of the evidence for a role for noradrenaline in schizophrenia reviewed by Hornykiewicz relates to the localization of noradrenaline in limbic 'emotional' parts of the brain and behavioural evidence indicating that noradrenaline is involved in stress responses. Though this fits a role for noradrenaline in schizophrenia, it is also consistent with noradrenaline involvement in affective disorders, where the evidence is in fact stronger: of course, evidence of a similar sort with comparable or stronger documentation applies as well for dopamine. In extensive studies of catecholamines in postmortem samples of human brain the concentrations of dopamine are found to be at least as high as, if not higher than, those of noradrenaline in several of the limbic forebrain areas in which Hornykiewicz indicates that noradrenaline is predominant (for example, amygdala, nucleus accumbens). A sub-dissection of the nucleus accumbens, as defined by Brockhaus ${ }^{2}$, has failed to reveal an area in which noradrenaline is present in excess over dopamine.

As for specific abnormalities of noradrenaline or dopamine or their synthetic or degrading enzymes or receptors in schizophrenic brain, there are published data with regard to both catecholamines. Changes have been found in levels of dopamine and noradrenaline and/or their metabolites in schizophrenic brain or spinal fluid. However, on balance the changes have been modest and not consistently reproduced in different laboratories. Moreover, in the great majority of instances it has not been possible to rule out the strong likelihood that observed changes are due to treatment with neuroleptics. In the case of the very consistently observed increase in number of dopamine receptors in schizophrenic brain $^{3.4}$, there is evidence that the changes are related to neuroleptic drug treatment ${ }^{5}$. Furthermore, one of us (G.R.) has found recently that significantly increased levels of noradrenaline (and dopamine) in cerebrospinal fluid from patients with paranoid schizophrenia $(n=28)$ were seen only in those patients who were receiving neuroleptic medication (about half) and the noradrenaline levels were independent of psychiatric ratings ${ }^{6}$.

Thus, there are no reproducible changes in postmortem brain indicating that either noradrenaline or dopamine is causally involved in the symptoms of schizophrenia. What is impressively established is that dopamine is involved in the influences of drugs on schizophrenic symptoms. The therapeutic efficacy of neuroleptics is closely correlated with their relative potencies in blocking one subtype of dopamine receptor, the $D_{2}$ receptor ${ }^{7,8}$. Hornykiewicz points out that numerous neuroleptics are potent $\alpha$-adrenergic receptor blockers. While this is true and while some neuroleptics are also very potent muscarinic anticholinergic, antihistamine and antiserotonin drugs, there is no correlation between their potencies in blocking any of these other receptors and therapeutic effects $^{9}$. Moreover it is unlikely that the low parkinsonism-inducing potency of neuroleptics such as thioridazine is associated with noradrenaline receptor blockade. Thioridazine is only as active an $\alpha$-adrenergic antagonist as the principal neuroleptics mentioned above, and its unusually potent anticholinergic actions probably account for the low incidence of extrapyramidal side effects ${ }^{10}$.

The mediation of neuroleptic therapeutic effects by dopamine receptor blockade is probably as well established as is the mechanism of action for any group of drugs in psychiatry and for that matter in general medicine. Amphetamines, which act by releasing catecholamines, predominantly dopamine, dramatically and selectively worsen schizophrenic symptoms in some patients ${ }^{11}$. Dopamine is more likely to be involved than noradrenaline, since L-dopa, which is converted in the brain largely into dopamine with little if any noradrenaline formed, also exacerbates schizophrenic symptoms ${ }^{12}$. Thus, one can titrate schizophrenic symptoms up or down by modulating synaptic levels of dopamine. This by no means implies a causal role for dopamine in schizophrenia. However, it does suggest that dopamine systems may be fairly closely linked to whatever is fundamentally aberrant in schizophrenic brain. Future effort needs to be directed to understanding the nature of the relationship between dopamine and other modulatory neuronal systems in brain, a relationship which one would expect to be altered by neuroleptic drugs.

L.L. Iversen is at the Neuroscience Research Centre, Merck, Sharp and Dohme Ltd, Hertford Road, Hoddesdon, Hertfordshire ENII $9 B U ;$ G.P. Reynolds is at the MRC Neurochemical Pharmacology Unit Brain Tissue Bank, Addenbrooke's Hospital, Cambridge $C B 22 Q Q$, and S.H. Snyder is at the Department of Neuroscience, Johns Hopkins Medical School, 725 North Wolf St, Baltimore, Maryland 21205.

1. Hornykiewicz, O. Nalure 299, 484 (1982)

2. Brockhaus, H. J. Physiol. Neurol. 51, 1 (1942)

3. Owen, F. et al. Lancet ii, 223 (1978).

Lee, T. el al. Nature 274, 897 (1978).

Mackay, A.V.P. et al. Arch. gen. Psychiat. 39, 991 (1982)

6. Gaitaz, W.F. et al. Psychiat. Res. 8, 243 (1083).

Creese, I. Burt, D.R. \& Snyder, S.H. Science 192. $481(1976)$.

Sceman, P. el al. Nature 261, 717 (1976).

9. Perculka, S.J. \& Snyder, S.H. Am. J. Psychiat. 137, $1518(1980)$.

10. Miller, R.J. \& Hiley, C.R. Nature 248, 596 (1974)

11. Janowsky, D.A. \& Davis, J.M. Arch. gen. Psychiat. 33, 304 (1976).

12. Angrist, B., Sathananthan, (i. \& Gershon, S. Psychopharmacologia 31, 1 (1973).

\section{Oleh Hornykiewicz replies:}

I AM pleased that Iversen et al. make such a strong case for the need of discriminating between the role of brain dopamine receptor blockade in the antipsychotic activity of the antidopominergic neuroleptics which no one would dispute - and the rather hypothetical role of dopamine in the pathophysiology of schizophrenia. The introductory paragraphs of my commentary were actually aimed at cautioning against this grave mistake. Strangely, despite their well founded warning against the misuse of the 'drug evidence', towards the end of their communication Iversen et al. come close to falling victim to this mistake by attaching so much weight to the fact that in schizophrenic patients, "one can titrate schizophrenic symptoms up or down by modulating synaptic levels of dopamine" with drugs such as amphetamine or L-dopa. Although Iversen et al. are swift to add that this "by no means implies a causal role for dopamine in schizophrenia", to contemplate such drug effects in isolation from other evidence is not very helpful to future research. As I have already explained one also can titrate Parkinsonian (and many other) symptoms up or down by modulating the levels of brain acetylcholine with cholinergic drugs, but it would not have resulted in any 